
Political and Social Research

\title{
Integrated Postsecondary Education Data System (IPEDS): Fall Staff, 1987
}

\author{
U.S. Dept. of Education \\ National Center for Education Statistics, \\ and Equal Employment Opportunity Commission \\ ICPSR 9529
}

